\title{
Modifications in anesthesia techniques and safety protocols at the outset of the COVID-19 pandemic, in a tertiary care center
}

Sangeeta Vivek Shetty MD, DNB*, Vijay L Shetty MD, Gita D Baria MD, Nishigandha G Sawant DNB, Hitesh Rajendra Singhavi MDS Department of Anaesthesia, Fortis Hospitals, Mulund Goregaon Link Road, Mulund, Mumbai.

*Corresponding Author: Sangeeta Vivek Shetty, Department of Anaesthesia, Fortis Hospitals, Mulund Goregaon Link Road, Mulund, Mumbai.

Received date: February 13, 2021 : Accepted date: April 20, 2021 : Published date: April 23,2021

Citation: Sangeeta V Shetty, Vijay L Shetty, Gita D Baria, Nishigandha G Sawant, Hitesh R Singhavi. (2021) Modifications in anesthesia techniques and safety protocols at the outset of the COVID-19 pandemic, in a tertiary care center. Journal of Clinical Surgery and Research. 2(2) DOI: $10.315792768-2757 / 006$

Copyright: (92021 Sangeeta Vivek Shetty, This is an open-access article distributed under the terms of the Creative Commons Attribution License, which permits unrestricted use, distribution, and reproduction in any medium, provided the original author and source are credited.

\section{Abstract}

Background and Aims: Anaesthesiologists, being involved in a variety of aerosol generating procedures during daily practice, are rendered especially vulnerable to the COVID-19 virus.

Our unit, situated in India's epicenter, is managing both Covid and non Covid patients. The aim of this retrospective study was to analyse the impact of modifications in anaesthesia techniques and safety protocols at the outset of the COVID-19 pandemic.

Methods: We reviewed anaesthesia charts of 454 consecutive patients requiring anaesthesia services between 25 March 2020 and 31 May 2020. An anonymous survey was carried out by circulating a questionnaire among the 26 Anaesthesiologists of the Department using an android application.

Results: Anaesthesia technique modifications were performed in 55.7\% of the studied cohort. Modifications included, no mask ventilation, use of rapid sequence intubation, shutting the carrier gas flows at breathing circuit disconnection (45.6\%), use of intubation box (32.2\%), conversion of GA to SA (11.0\%), use of plastic sheet (7.7\%), use of L screen for SA $(0.9 \%)$.

Skill limitation due to PPE and discomfort using intubation box occurred in $84.61 \%$ and $53.85 \%$ anaesthesiologists respectively. $18.5 \%$ anaesthesiologists developed symptoms, however none of them tested RT-PCR positive. No anaesthesiologist required isolation or hospitalization due to COVID-19 infection.

Conclusions: The Lockdown period at the outset of the COVID-19 pandemic enabled strict triage and transmission prevention practices to be introduced and practiced. None of the anaesthesiologists in our department required hospitalisation or quarantine due to COVID-19.

Key Words: anesthesia; covid-19; sars-cov-2; operating rooms; safety management; aerosols; personal protective equipment

\section{Introduction}

Once the pandemic of COVID-19 was declared by the World Health Organisation (WHO) a nationwide lockdown was implemented to prevent the community spread between March $25^{\text {th }}$ to May $31^{\text {st }} 2020$. It followed the initial reports of the rampage caused by the Severe Acute Respiratory Syndrome-Corona Virus-2 (SARS-CoV-2) in health care facilities of the most developed nations of the world. As of December 2020, 6,52,57,767 cases and 15,13,179 deaths have occurred worldwide. In India, 96,08,211 cases and 1,39,700 deaths have been reported [1].

Anaesthesiologists are involved in a variety of aerosol generating procedures during a typical working day [2]. The vulnerability to the virus resulted in anaesthesia and intensive care to be classified as high-risk specialities [3]. Various guidelines have been suggested by national and international anaesthesia societies for the safety of healthcare workers $[4$, 5].

Our hospital, a corporate tertiary referral centre started admitting COVID19 patients as per local Government (Brihanmumbai Municipal Corporation) policy since the outset of the pandemic. It is situated in Mumbai city which has been declared the epicentre of India's corona virus outbreak, accounting for more than a fifth of the total case count. Being Joint Commission International (JCI) and National Accreditation Board of Hospitals (NABH) accredited standard practices of hygiene and infection control were already maintained at the workplace. However, we 
quickly realised that constant mindfulness of each action would be our only armamentarium and the way forward.

There being no definitive treatment available, we studied the impact of modifications in anaesthesia techniques and operation theatre safety protocols implemented by our unit at the onset of the COVID-19 pandemic.

\section{Methodology}

This single centre, retrospective study was approved by the Institutional Ethical Committee. (Ref: IEC 2020/OAS/20). The requirement for informed consent was waived under the regulations of the committee.

Anaesthesia charts of consecutive outpatients and admitted patients requiring anaesthesia services during the period of lockdown between March $25^{\text {th }}$ to May $31^{\text {st }} 2020$ were reviewed. Data pertaining to the type of procedure performed, type of anaesthesia given, pre-operative real time reverse transcription polymerase chain reaction (rRT-PCR) test status of the patient were collected. Suspected Covid positive patients included untested patients as well as those patients whose throat swab had been collected for rRT-PCR analysis but did not have the test results preoperatively. Modification of anaesthesia technique constituted the use of intubation box or plastic sheet, conversion of procedures normally performed under general anaesthesia (GA) or total intravenous anaesthesia (TIVA) to spinal anaesthesia (SA) and others (no mask ventilation, use of rapid sequence intubation, shutting the carrier gas flows whenever breathing circuit disconnected from the patient.)

26 Anaesthesiologists comprising 15 consultants and 11 registrars who were working full time in the department (in Covid and non Covid areas of the hospital) during the study period were asked to complete a questionnaire. This was done anonymously via SurveyMonkey (Google) application and forwarded to the participants via android mobile.

The primary outcome was to estimate the need of anaesthesiologists to visit COVID-19 fever clinic, undergo isolation, or hospitalization.

\section{Statistical analysis -}

Categorical data was analyzed using chi-square test. A two-sided $\mathrm{P}$ value $<0.05$ was considered statistically significant. Analyses were performed using SPSS version 20 (SPSS, Inc., Chicago, IL, USA).

\section{Results}

The analysis was done for 454 patients. Anaesthesia technique modification was performed in $55.7 \%$ of the studied cohort. $42.3 \%$ cases were done under GA with ETT, least number of cases were done using LMA (3.3\%).

In the operation theatre, maximum modification was done for cardiac surgery (100\%) and least for obstetric and gynaecology procedures $(26.7 \%)$. The most common type of anaesthesia modification was avoidance of mask ventilation, use of rapid sequence intubation, shutting the carrier gas flows whenever breathing circuit was disconnected from the patient $(45.6 \%)$. This was followed by use of intubation box $(32.2 \%)$, conversion of GA to SA (11.0\%), use of plastic sheet (7.7\%), use of L screen for SA $(0.9 \%)$. Plastic surgery, neurosurgery and obstetrics and gynaecology were the specialities with the most COVID suspect patients.

In non-operating room areas, all brachytherapy cases had conversion of technique from GA to SA. Although majority of cases were done in endoscopy, no modifications in technique was possible. All cases in endoscopy and brachytherapy had RT PCR results available before they were taken up.

$18.5 \%$ anaesthesiologists from the department had symptoms related to COVID, however, none tested positive or required hospitalisation. 1 consultant was in self-isolation as a close contact had returned from overseas. All anaesthesiologists from the department, were motivated to come to work in spite of the fear of getting infected.

\section{Discussion}

Following the declaration of lockdown, the work pattern in the hospital was continuously evolving. Triage at entry points allowed restricted entry to the hospital or diversion to the Covid fever clinic. Green stickers to wear indicated symptom free state, thus permission to enter. A revised standard operating procedure document incorporated the modifications in requirements of anaesthesia. Part telephonic pre anaesthesia consultations were encouraged in an attempt to reduce physical contact with the patient. Maintaining communication with patients while still avoiding close contact, presented a clinical and ethical dilemma.

In our analysis of the modifications of Anaesthesia, we noted that all patients receiving General Anaesthesia (GA) had implemented the suggested protocols of adequate pre oxygenation with face mask, rapid sequence intubation and mandatory shutting off of fresh gas flows whenever intubation was undertaken and when the circuit was disconnected [6]. This was a result of simulations for conduct of GA with mannequins which was mandatory for all anaesthesia technicians and anaesthetists alongside the Personal Protective Equipment (PPE) training in the early part of the pandemic [7].

All airway interventions, were treated as a potential risk for transmission. Acrylic intubation boxes or plastic sheets were available as barriers for transmission of aerosols generated [8]. More than half of the anaesthesiologists had difficulty using the intubation box. There was a learning curve owing to the altered ergonomics, hampered vision due to fogging and reflection of light on multiple layers of plastic. Begley et al have shown that aerosol boxes may paradoxically increase the risk to clinicians involved in COVID-19 airway management [9]. Rosenblatt et al have also questioned the use of the same [10].

The use of Laryngeal Mask Airways (LMAs) during the pandemic was very low with only 15 out of 263 cases conducted under GA being done with the LMA. (Table1.) Concerns have been expressed over the possible air leaks as well as requirement of adjustments of the device which in turn necessitates proximity to a possible source of infection [11]. The evidence for use of LMA in COVID is limited. Yang et al have recommended its use in an airway rescue scenario over face mask ventilation before and in between attempts at laryngoscopy [12]. Other authors have not shared these concerns and have advised regular use of LMAs due to their advantages over general anaesthesia with endotracheal tube [13].

\begin{tabular}{|c|c|}
\hline Anaesthesia & Number (Percentage) \\
\hline General Anaesthesia (ETT) & $192(42.3 \%)$ \\
\hline General Anaesthesia (LMA) & $15(3.3 \%)$ \\
\hline Regional Anaesthesia & $110(24.3 \%)$ \\
\hline TIVA & $56(12.3 \%)$ \\
\hline MAC with Sedation & $21(4.6 \%)$ \\
\hline MAC & $60(13.2 \%)$ \\
\hline Total & $454(100 \%)$ \\
\hline
\end{tabular}


Table 1: Type of Anaesthesia used
TIVA $=$ Total Intravenous Anaesthesia
MAC $=$ Monitored Anaesthesia Care
$\mathrm{ETT}=$ Endo tracheal tube
LMA = Laryngeal Mask Airway

\begin{tabular}{|l|l|l|l|}
\hline Surgical Speciality & $\begin{array}{l}\text { Number Cases } \\
\text { (\% of total) }\end{array}$ & $\begin{array}{l}\text { Anaesthesia } \\
\text { Modification done }\end{array}$ & $\begin{array}{l}\text { Suspected COVID } \\
\text { positive patients }\end{array}$ \\
\hline Oncology & $78(17.2 \%)$ & $73(93.6 \%)$ & $6(7.7 \%)$ \\
\hline Urology & $61(13.4 \%)$ & $42(68.9 \%)$ & $9(14.7 \%)$ \\
\hline $\begin{array}{l}\text { Obstetrics and } \\
\text { Gynaecology }\end{array}$ & $60(13.2 \%)$ & $17(28.3 \%)$ & $23(38.3 \%)$ \\
\hline General Surgery & $53(11.7 \%)$ & $44(83.0 \%)$ & $14(26.4 \%)$ \\
\hline Ortho & $25(5.5 \%)$ & $17(68 \%)$ & $5(20 \%)$ \\
\hline Neurosurgery & $21(4.6 \%)$ & $20(95.2 \%)$ & $9(42.8 \%)$ \\
\hline Plastic Surgery & $14(3.08 \%)$ & $11(78.6 \%)$ & $7(50.0 \%)$ \\
\hline Cardiac & $13(2.9 \%)$ & $13(100 \%)$ & $4(30.8 \%)$ \\
\hline Spine & $12(2.6 \%)$ & $9(75.0 \%)$ & $2(16.7 \%)$ \\
\hline Vascular & $6(1.3 \%)$ & $3(50.0 \%)$ & $1(16.7 \%)$ \\
\hline $\begin{array}{l}\text { Non-Operating Room } \\
\text { Procedures }\end{array}$ & & & 0 \\
\hline Endoscopy & $56(12.3 \%)$ & 0 & $6(12.2 \%)$ \\
\hline Cath lab & $49(10.8 \%)$ & $9(18.4 \%)$ & 0 \\
\hline Brachytherapy & $6(1.3 \%)$ & $6(100 \%)$ & $86(18.9 \%)$ \\
\hline Total & $454(100 \%)$ & $253(55.7 \%)$ & \\
\hline
\end{tabular}

Our analysis also revealed that whenever appropriate for the surgery, regional anaesthesia was chosen over general anaesthesia (which would have been a choice in the pre-pandemic era) given its advantages of lesser aerosolization during intubation and extubation as well as accidental circuit disconnections $[14,15]$. Also, given the propensity for pulmonary involvement in the Covid patients, avoiding general anaesthesia could have pulmo-protective effects due to prevention of atelectasis and maintained ciliary function and pulmonary clearance.

Regional anaesthesia was used in $80 \%$ of obstetrics and gynaecology and $54 \%$ of urology patients. (Graph 1.) In short cases such as radiotherapy for cancer cervix (brachytherapy), short urosurgeries, spinal anaesthesia (SA) replaced GA. (Table 3.) The short duration of action of Chloroprocaine made it the drug of choice for spinal anaesthesia. After a 15 to 20minute period of observation in the operation theatre (OT), these patients were shifted to their respective wards. An L-shaped metal bar called the L-screen had been locally suggested to enable positioning of patients during neuraxial blockade and reduce the close contact of attendants with the patient. However, its use was not favoured in the department. Attempts were made to reduce the use of total intravenous anaesthesia (TIVA) thus preventing airway manipulation in case of deeper planes of sedation. However, Choksi et al favoured TIVA [16]. Use of TIVA could not be avoided in endoscopy patients.
Plastic surgery, Obstetrics and Gynaecology and Neurosurgery were the specialities with the highest number of suspected Covid patients as a result of their emergent nature. One obstetric patient in our cohort, tested RT-PCR positive postoperatively after undergoing an emergency caesarean section under SA uneventfully. All patients from endoscopy and brachy therapy had RT PCR results available pre procedure.

The anaesthesiologists were divided into two groups that worked alternately on weekly basis in the operating rooms. A third group contributed to rotas in emergency room, intensive care units (ICU) and Covid ICU. Restricting the operating list to urgent or semi-emergency surgeries made this division possible.

The drill of wearing basic personal protection formed a daily routine (Fig 1) In the first week standard 3 ply masks were used, thereafter, a set of five N95 National Institute for Occupational Safety and Health (NIOSH) certified masks were used in rotation, stored in paper bags and discarded after five uses per mask [17]. The previous SARS outbreak in 2003, suggested the use of positive airway pressure respirators (PAPR) along with N95 masks [18]. However, PAPR was not used in our setup. Maintaining interpersonal distance, long-sleeved cotton gown, frequent hand washing / sanitisation, double layered gloves for airway procedures were encouraged [19]. 


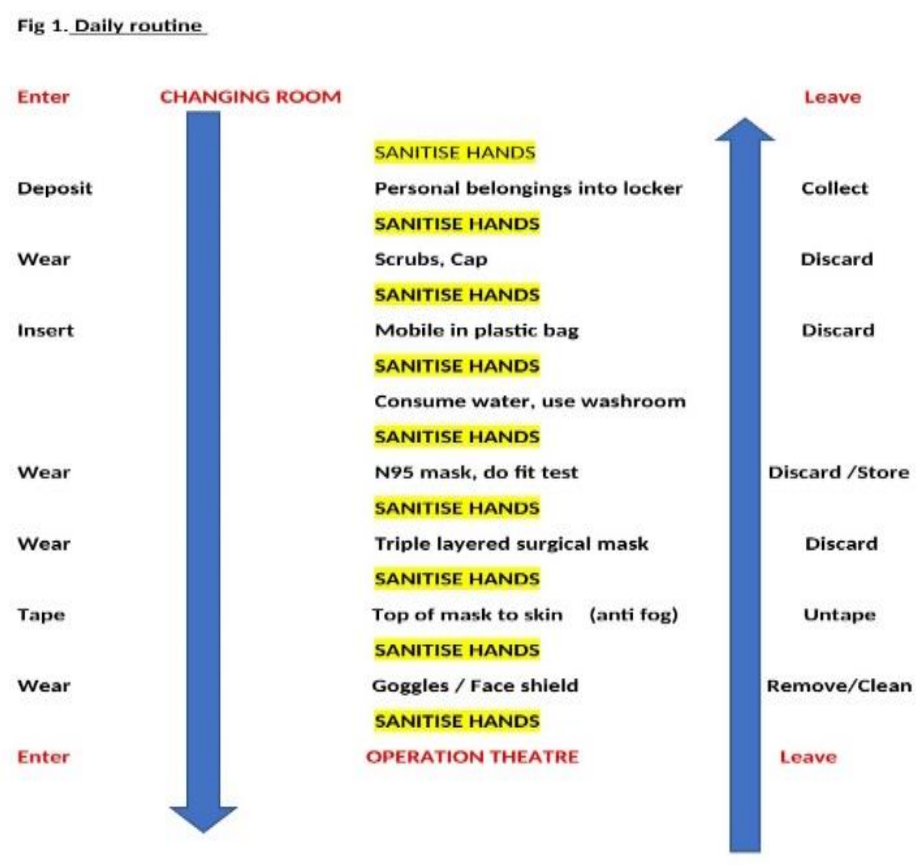

PPE was donned for all suspected cases. In our study, most anaesthesiologists experienced skill limitation due to PPE. Fogging, discomfort, restriction of movements, difficulty in communication, were limitations of PPE use. Repeated mask or goggle adjustment without prior hand sanitisation was discouraged. Donning and Doffing of PPE was assisted by dedicated nurse, signages on the OT walls and segregated sites [20].

Two adjacent OTs containing only the essential equipment were designated for COVID-19 positive or suspected patients. (Fig 2). Proximity to the entry area of the OT complex minimised contamination of the premises [21]. One of them had an automatic door opening system and served as a doffing area. Recommendations of conversion to negative pressure operating theatres have been made. [22, 23]. The endoscopy unit had a negative pressure room. However, operation theatres had positive pressure systems. Here, the heating ventilation and air conditioning (HVAC) units of the COVID-19 OTs were kept separate from the other theatres. The temperature was maintained between $20-22$ degrees Celsius and humidity at $55-60 \%$. High Efficiency Particulate Air (HEPA) filters with 25 room air exchanges per hour was the standard. Plastic sheets covered most surfaces including electrocautery, anaesthesia workstation and monitor. Necessary high alert medications and anaesthesia drugs were dedicated to the OT [24]. Once the patient entered, doors were shut. A runner (nurse) ready in PPE stationed outside the OT provided equipment, drugs, essential documentation or called for help if required.

Fig. 2 Schematic representation of Operation Theatre layout

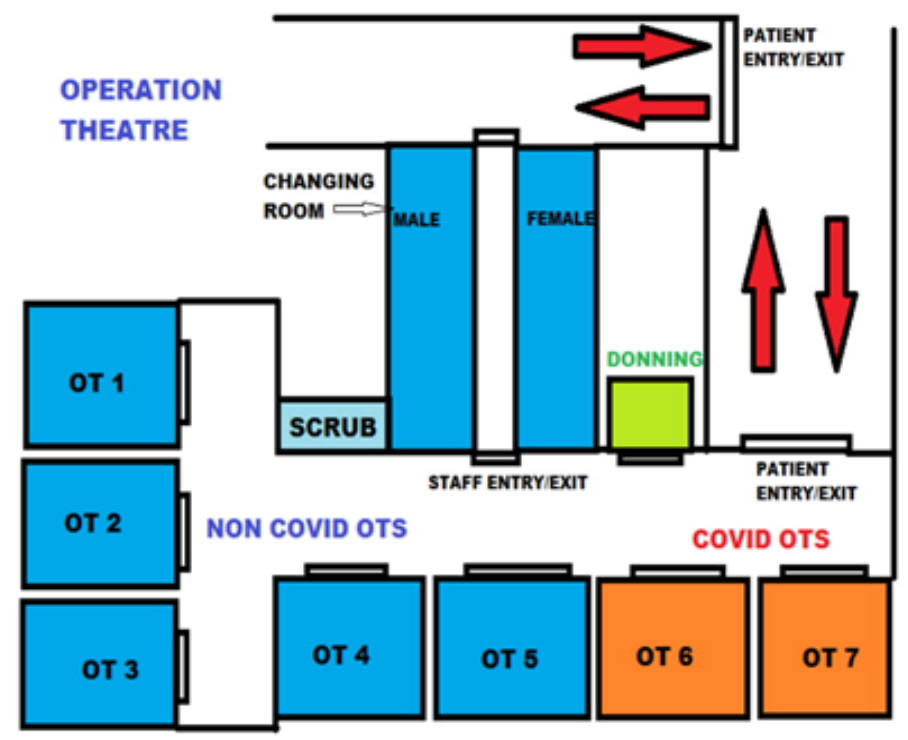


All awake patients came into the OT wearing a triple layered surgical mask. Protection during transfer included plastic sheet covers and heat and moisture exchange filters (HMEF) to minimise aerosolization in ventilated patients. Triage of surgical patients was maintained. (Fig.3)

\section{Fig 3. Triage of surgical patients}

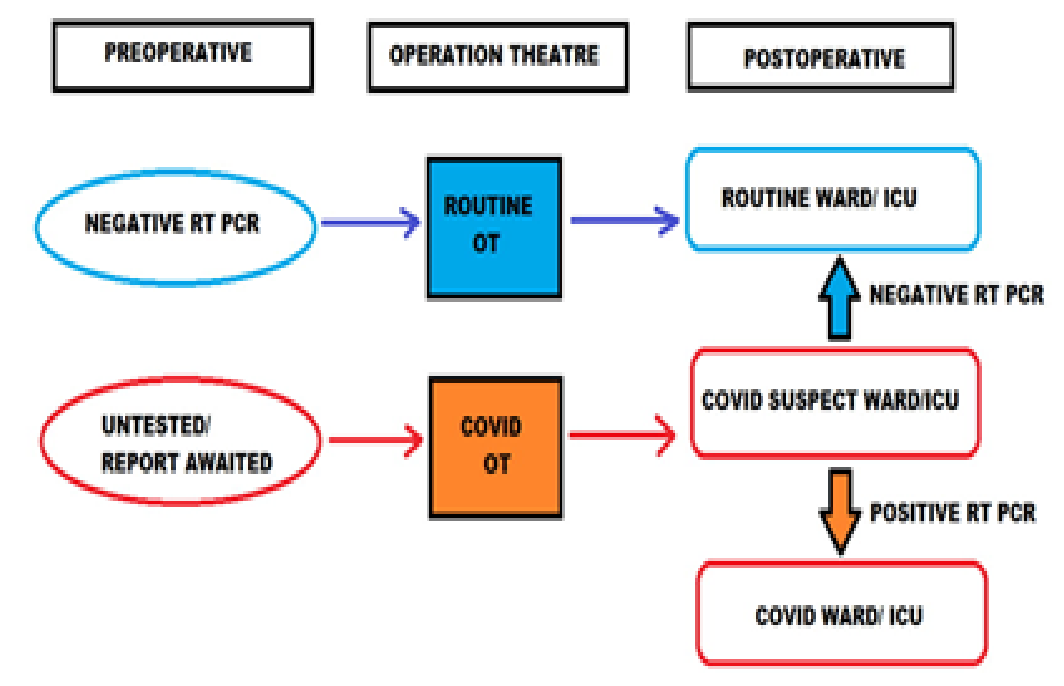

OTs were kept closed for 20 minutes after each suspected and positive case to allow for air exchanges before housekeeping staff entered for cleaning [25]. Instruments were sent to the central sterile services department (CSSD) in a separate pack. Floor, walls were cleaned with $1 \%$ hypochlorite, surfaces with $75 \%$ alcohol and fogging with vaporised hydrogen peroxide $[24,26]$. Manufacturer instructions were followed for video laryngoscope cleaning. Filters, breathing circuit, soda lime were changed for each case receiving general anaesthesia. Wherever possible, disposable material was used. Hospital protocol defined the collection of waste in bin liners. They were labelled - suspected or Covid positive and discarded at the end of the case as per biomedical waste management policy.

The dining area within the OT complex, remained one of the most difficult areas to enforce discipline. Reduction in the number of chairs limited the number of people present at a time. Diagonal seating or oneway facing seating was permitted.

OT and housekeeping staff were retrained regarding proper use of mask, importance of handwashing and maintaining interpersonal distance. Mock drills for donning, doffing, instructional videos and webinars on the subject were used as tools for the same

ICMR had recommended the use of Hydroxychloroquine (HCQ) prophylaxis for health care workers [27]. In our unit however, it was an individual choice, taken by $50 \%$ of the personnel.

Anaesthesiologists from the department who visited the Covid fever clinic (OPD) had fatigue as the commonest symptom. None of them tested RTPCR positive. One anaesthesiologist had self-isolated due to close contact with a family member who had travelled overseas. No anaesthesiologist required isolation or hospitalisation due to COVID -19 infection.

In spite of the lurking fear of getting infected and carrying the virus home, all the department members were committed to their work. Positive reenforcement and working in high-risk zones by example went a long way in encouraging the team members to continue the smooth functioning of the department and ICU areas of the hospital. Anaesthesiologists thus played a dual role in managing COVID ICU and COVID suspected patients in operation theatre.

\section{Conclusion}

The Lockdown period enabled new practices to be tested and personnel became accustomed to them. Staff were trained, drills could be carried out. Stringent hygiene techniques and triage at each level became the pillars of support. None of the anaesthesiologists in our department required hospitalisation or quarantine due to COVID-19.

With the lockdown easing off, the number of COVID-19 cases in the community is rising and we will be definitely encountering a higher number of infected cases. Further modification of protocols may be required to accommodate increasing OT lists and anaesthesia personnel requirements in other areas of the hospital.

Modifications in anaesthesia techniques and safety protocols have become the new normal. They have kept us in good stead so far and we must not let our guard down. These practices may be considered as a model for other Covid-care setups and similar contingencies in the future.

\section{Funding source - Nil}

Acknowledgement - Would like to thank Dr Vaishali Shende for data collection and Dr

Hemalata Iyer for critical review of the manuscript.

Presented as part of meeting / publication - No

Registration number in case of clinical trial - Not applicable

\section{Conflicts of interest - Nil}

\section{Criteria for inclusion in authors list}

Dr Sangeeta Shetty - Concept, design, definition of intellectual content, literature search, manuscript preparation.

Dr Vijay Shetty - Definition of intellectual content, manuscript editing, manuscript review. 
Dr Gita Baria - Design, data acquisition, data analysis, manuscript editing.

Dr Nishigandha Sawant - Literature search, manuscript editing, manuscript review.

Dr Hitesh R Singhavi - Statistical analysis, manuscript editing, manuscript review.

Manuscript has been read by all authors - Yes as mentioned in Contributors form

\section{References}

1. WHO Coronavirus Disease (COVID-19) Dashboard

2. Harding H, Broom A, Broom J. (2020) Aerosol generating procedures and infective risk to healthcare workers from SARSCoV-2: the limits of the evidence. Journal of Hospital Infection. 105:717-725.

3. Cook TM. (2020) Risk to health from COVID-19 for anaesthetists and intensivists - a narrative review. Anaesthesia.

4. Zheng H, Hébert HL, Chatziperi A, Meng W, Smith BH, Yan J, Zhou Z, Zhang X, Luo A, Wang L, Zhu W, Hu J, Colvin LA. (2020) Perioperative management of patients with suspected or confirmed COVID-19: review and recommendations for perioperative management from a retrospective cohort study, British Journal of Anaesthesia.

5. T. M. Cook, K. El-Boghdadly, B. McGuire, A. F. McNarry, A. Patel and A Higgs. (2020) Consensus guidelines for managing the airway in patients with COVID-19 Guidelines from the Difficult Airway Society, the Association of Anaesthetists the Intensive Care Society, the Faculty of Intensive Care Medicine and the Royal College of Anaesthetists Anaesthesia, 75, 785799.

6. Velly et al. (2020) Anaesth Crit Care Pain Med 39; 395-415 Guidelines: Anaesthesia in the context of COVID-19 pandemic.

7. Dieckmann, P., Torgeirsen, K., Qvindesland, S.A. et al. (2020) The use of simulation to prepare and improve responses to infectious disease outbreaks like COVID-19: practical tips and resources from Norway, Denmark, and the UK. Adv Simul 5, 3 .

8. Canelli R, Connor CW, Gonzalez M, Nozari A, Ortega R. (2020) Barrier enclosure during endotracheal intubation. N Engl J Med.

9. Begley JL, Lavery KE, Nickson CP, Brewster DJ. (2020) The aerosol box for intubation in coronavirus disease 2019 patients: an in-situ simulation crossover study. Anaesthesia.

10. Rosenblatt WH, Sherman JD. (2020) More on Barrier Enclosure during Endotracheal Intubation N Engl J Med.

11. Lim WY, Wong P. (2020) Supraglottic airways in the management of COVID-19 patients. Anaesth Crit Care Pain Med.

12. Yang W-S, Hou S-W, Lee B-C, Chiang W-C, Chien Y-C, Chen S-Y, et al. (2020) TaipeiAzalea - Supraglottic airways (SGA) preassembled with high-efficiency particulate air (HEPA) filters to simplify prehospital airway management for patients with outof-hospital cardiac arrests (OHCA) during coronavirus disease 2019(COVID-19) pandemic. Resuscitation. 151:3-5.
13. Sorbello M, El-Boghdadly K, Di Giacinto I, Cataldo R, Esposito C, Falcetta S, et al. (2020) The Italian coronavirus disease 2019 outbreak: recommendations from clinical practice. Anaesthesia. 75:724-732.

14. Brewster DJ, Chrimes NC, Do TBT, Fraser K, Groombridge CJ, Higgs A, et al. (2020) Consensus statement: Safe Airway Society principles of airway management and tracheal intubation specific to the COVID-19 adult patient group. Med J Aust.

15. Lie SA, Wong SW, Wong LT, Wong TGL, Chong SY. (2020) Practical considerations for performing regional anesthesia: lessons learned from the COVID-19 pandemic. Can J Anesth. 67:885-892.

16. Chokshi T, Channabasappa S, Vergheese DC, Bajwa SJ, Gupta B, Mehdiratta L. (2020) Re-emergence of TIVA in COVID times. Indian J Anaesth. 64:125-133.

17. Centers for Disease Control and Prevention. Pandemic planning: Recommended guidance for extended use and limited reuse of N95 filtering facepiece respirators in healthcare settings.

18. Wax RS, Christian MD. (2020) Practical recommendations for critical care and anesthesiology teams caring for novel coronavirus (2019-nCoV) patients. Can J Anaesth. 67(5):568576.

19. Chen XD, Shang Y, Yao SL, et al. (2020) Perioperative care provider's considerations in managing patients with the COVID19 infections. Transl Perioper Pain Med. 7(2)216-214.

20. Chughtai AA, Chen X, Macintyre CR. (2018) Risk of selfcontamination during doffing of personal protective equipment. Am J Infect Control. 46(12):1329-1334.

21. Coccolini et al. (2020) Surgery in COVID-19 patients: operational directives. World Journal of Emergency Surgery. 15(25).

22. Ong SWX, Tan YK, Chia PY, et al. (2020) Air, surface environmental and personal protective equipment contamination by severe acute respiratory syndrome coronavirus 2 (SARSCoV-2) from a symptomatic patient. JAMA.

23. Chow TT, Kwan A, Lin Z, Bai W. (2006) Conversion of operating theatre from positive to negative pressure environment Journal of Hospital Infection. 64, 371-378.

24. Malhotra N, Bajwa SJ, Joshi M, Mehdiratta L, Trikha A. (2020) COVID Operation Theatre- Advisory and Position Statement of Indian Society of Anaesthesiologists (ISA National). Indian J Anaesth. 64:355-362.

25. Bajwa SJ, Sarna R, Bawa C, Mehdiratta L. (2020) Peri-operative and critical care concerns in coronavirus pandemic. Indian $\mathbf{J}$ Anaesth. 64: 267-274.

26. Kampf G, Todt D, Pfaender S, Steinmann E. (2020) Persistence of coronaviruses on inanimate surfaces and their inactivation with biocidal agents. J Hosp Infect. 104: 246-251.

27. National Task Force for COVID-19 in India. (2020) Recommendation for empiric use of hydroxychloroquine for prophylaxis of SARS-CoV-2 infection. 